\title{
Strain Development and Damage Accumulation Under Ion Irradiation of Polycrystalline Ge-Sb-Te Alloys
}

\author{
S. Privitera ${ }^{1, *}$, A. M. Mioํ, W. Zhang², R. Mazzarello ${ }^{3}$, C. Persch ${ }^{4}$, M. Wuttig ${ }^{4}$, and E. Rimini ${ }^{1}$ \\ ${ }^{1}$ IMM-CNR, Zona Industriale VIII Strada 5, 95121 Catania, Italy \\ ${ }^{2}$ Center for Advancing Materials Performance from the Nanoscale, State Key Laboratory for \\ Mechanical Behavior of Materials, Xi'an Jiaotong University, Xi'an 710049, P. R. China \\ ${ }^{3}$ Institute for Theoretical Solid State Physics, JARA-FIT and JARA-HPC, RWTH Aachen University, Aachen, Germany \\ ${ }^{4}$ I. Physikalisches Institut (IA) and JARA-FIT, RWTH Aachen University, Sommerfeldstraße 14, 52074 Aachen, Germany
}

\begin{abstract}
The atomic displacement produced by ion irradiation with $150 \mathrm{keV} \mathrm{Ar}^{+}$ions has been studied in $\mathrm{Ge}_{1} \mathrm{Sb}_{2} \mathrm{Te}_{4}$ and $\mathrm{Ge}_{2} \mathrm{Sb}_{2} \mathrm{Te}_{5}$. Electrical, optical and structural measurements have been employed to characterize the induced electrical and structural modifications. At low temperature the amorphization threshold, evaluated by in situ reflectivity measurements, is independent of the composition and the crystalline structure, and it is equal to $1 \times 10^{13} \mathrm{~cm}^{-2}$. At room temperature, at which dynamic annealing can take place, $\mathrm{Ge}_{2} \mathrm{Sb}_{2} \mathrm{Te}_{5}$ and $\mathrm{Ge}_{1} \mathrm{Sb}_{2} \mathrm{Te}_{4}$ in the rocksalt phase exhibit the same amorphization threshold $\left(3 \times 10^{13} \mathrm{~cm}^{-2}\right)$. In the trigonal structure, instead, a higher fluence is required to amorphize the $\mathrm{Ge}_{1} \mathrm{Sb}_{2} \mathrm{Te}_{4}$, compared to $\mathrm{Ge}_{2} \mathrm{Sb}_{2} \mathrm{Te}_{5}$. The observed differences between the two compositions can be explained considering the effect of dynamic annealing during ion irradiation of the trigonal phase, which is characterized by the presence of van der Waals gaps. These may act as a preferential sink for the diffusion of the displaced atoms and the filling of these gaps tunes the electronic and structural properties. Filling of about $30 \%$ of the gaps produces an electronic transition from metallic to insulating behavior. By further increasing the disorder and filling more than $70 \%$ of the gaps the films convert into the rocksalt phase.
\end{abstract}

\section{Keywords:}

\section{INTRODUCTION}

Ion implantation with energetic ions leads to strain production due to the displacement of atoms from their lattice positions, thanks to the energy transfer from the projectile ions to the lattice atoms. At low temperatures, depending on the ion fluence, the strain can induce complete loss of the crystalline order with the production of an amorphized layer. At higher temperatures, where point defects are sufficiently mobile, recombination of self interstitial and vacancies produced in the collision cascades anneals out part of the produced damage (dynamic annealing) and, depending on the temperature, the amorphous layer can no longer be created, even after implantation at high fluences. ${ }^{1}$

Phase change materials, and in particular $\mathrm{Ge}-\mathrm{Sb}-\mathrm{Te}$ (GST) alloys belonging to the GeTe- $\mathrm{Sb}_{2} \mathrm{Te}_{3}$ pseudo-binary line, are characterized by rapid switching between the amorphous and the crystalline phase and by high electrical and optical contrast. ${ }^{2}$ The reversible fast transition

*Author to whom correspondence should be addressed. from the amorphous to the crystalline structure can be induced by electric or laser pulses. These properties have made phase change materials very attractive for applications as optical storage media (rewritable DVD) and to replace Flash memories for electronic data storage. ${ }^{3-6}$

Among the others, $\mathrm{Ge}_{2} \mathrm{Sb}_{2} \mathrm{Te}_{5}$ (GST225) is the most studied composition, since it allows the best performance in terms of speed and scalability. ${ }^{7}$ The stable crystalline structure of GST225 is trigonal, with space group (P-3m1), and it is formed at temperatures above $250{ }^{\circ} \mathrm{C} .{ }^{8,9}$ However, a metastable phase with rocksalt structure (space group Fm-3m) is obtained by annealing of an amorphous film at temperature between 130 and $200{ }^{\circ} \mathrm{C}$. In this structure, Te atoms occupy the anion sublattice, while the cation sublattice is shared by $\mathrm{Ge}, \mathrm{Sb}$ atoms and lattice vacancies. ${ }^{8}$ Indeed, since the number of Te atoms exceeds the number of $\mathrm{Ge}$ and $\mathrm{Sb}$ atoms there is a large amount of structural vacancies. For the composition GST225 20\% of cation sites are empty. The random distribution of vacancies in the cation sublattice of the rocksalt structure is common also to other GST stoichiometries, such 
as $\mathrm{Ge}_{1} \mathrm{Sb}_{2} \mathrm{Te}_{4}$ (GST124) and $\mathrm{Ge}_{3} \mathrm{Sb}_{2} \mathrm{Te}_{6}$, characterized by $25 \%$ and $16.7 \%$ of empty cation sites, respectively. These vacancies are often called stoichiometric vacancies, since they are governed by the film stoichiometry. ${ }^{10}$ It is important to note that phase change materials such as GST124 even contain further vacancies, which make these compounds metallic and lead to charge carrier concentrations of about $10^{20} \mathrm{~cm}^{-3}$ and $p$-type conductivity. The vacancies are sometimes called excess vacancies to differentiate them from the stoichiometry vacancies discussed above. ${ }^{11}$

The stable trigonal phase is instead a layered structure, consisting of stacked atomic plane sequences arranged along the hexagonal $c$-axis. ${ }^{7,8}$ According to the structure proposed by Matsunaga, ${ }^{8}$ based on X-ray diffraction analyses, the sequence of the layers in the trigonal phase is $\mathrm{Te}-\mathrm{Sb} / \mathrm{Ge}-\mathrm{Te}-\mathrm{Ge} / \mathrm{Sb}-\mathrm{Te}-\mathrm{Sb} / \mathrm{Ge}-\mathrm{Te}$, taking into account the presence of $\mathrm{Ge} / \mathrm{Sb}$ intermixing in the cation layers. The two adjacent Te planes are weakly bound by van der Waals forces, giving rise to relatively large separations (so called van der Waals gaps). ${ }^{12}$

Structures with pure $\mathrm{Ge}$ and $\mathrm{Sb}$ planes have also been proposed by Petrov ${ }^{13}$ (in which the Te atoms at the van der Waals gaps are bonded with Ge atoms only) or Kooi and De Hosson $(\mathrm{KH})^{7}$ (with Te atoms at the gap bonded with $\mathrm{Sb}$ atoms only). The latter structure is obtained by intercalating GeTe bilayer units into $\mathrm{Sb}_{2} \mathrm{Te}_{3}$ units. This effect has been first reported in superlattice structures. ${ }^{14,15}$ Subsequently, it has been shown to be a typical building mechanism for GeSbTe alloys, not related to an initial $\mathrm{GeTe} / \mathrm{Sb}_{2} \mathrm{Te}_{3}$ superlattice arrangement. ${ }^{16}$

It is known that the electrical properties of the crystalline structures of GST alloys are very sensitive to the degree of order. Indeed, it has been experimentally observed that, by annealing GeSbTe alloys at different temperatures, it is possible to modify the degree of disorder and even induce an Anderson insulator-to-metal transition. ${ }^{17,18}$ Recently, it has been shown that the ordering of the vacancies is the microscopic origin of this transition. ${ }^{19,20}$ According to theoretical simulations, in the disordered rock-salt phase, obtained at low annealing temperature, the electronic states at the Fermi energy are localized inside vacancy clusters. Thermal annealing at higher temperatures dissolves these clusters and orders the vacancies into planes, eventually leading to metallic behavior. ${ }^{19,20}$ Vacancy disorder has a pronounced impact on the thermal transport as well, modulating the thermal conductivity of GeSbTe alloys. ${ }^{21}$

The possibility to tune the disorder and to verify its effect by structural measurements is very attractive for many applications, such as the development of thermoelectric devices with high figure of merit ${ }^{22}$ and of temperatureindependent resistors. ${ }^{23}$ It is also of interest to realize phase change memories based on different degrees of order in the crystalline states, enabling multilevel data storage, with reduction of the RESET current ${ }^{24}$ and high temperature reliability.
In this paper we use ion irradiation with diluted cascades to induce atomic displacement in the crystalline phases of GST225 and GST124. We show that the produced strain modifies the optical and electrical properties. The amount of vacancies in the structure determines the stability window of the trigonal and cubic structures. The maximum degree of disorder that can be sustained by these structures is assessed.

\subsection{Sample Preparation}

Ion irradiation has been performed employing $150 \mathrm{keV}$ $\mathrm{Ar}^{+}$ions at different fluences in the range from $1 \times$ $10^{11} \mathrm{~cm}^{2}$ to $5 \times 10^{14} \mathrm{~cm}^{-2}$. The irradiation has been performed at room temperature and, for comparison, at $77 \mathrm{~K}$.

The irradiated samples were $50 \mathrm{~nm}$ thick films with composition GST225 or GST124, deposited on oxidized Si (100) wafers by DC sputtering at room temperature in the amorphous phase, and subsequently annealed at temperatures around $200{ }^{\circ} \mathrm{C}$ or $350{ }^{\circ} \mathrm{C}$, in order to obtain the rock-salt or the trigonal stable phase, respectively, as confirmed by XRD.

According to SRIM calculations, the projected range of $\mathrm{Ar}^{+}$ions is $90 \mathrm{~nm}$, assuring no $\mathrm{Ar}^{+}$ions stop into the GST film and the energy loss, both nuclear and electronic, is quite uniform across the film thickness. The sputtering yield for $\mathrm{Ge}, \mathrm{Sb}$ and $\mathrm{Te}$ for $\mathrm{Ar}$ at $150 \mathrm{keV}$ is about 0.3 atoms/ion. This means that, even in the case of the maximum adopted fluence $\left(5 \times 10^{14} \mathrm{~cm}^{-2}\right)$ less than 1 tenth of a monolayer is sputtered, and therefore this effect is negligible. The beam current was kept constant and equal to $30 \mathrm{nA} \mathrm{cm}^{-2}$.

\subsection{Experimental Details}

The modifications of the optical properties have been monitored in situ by measuring the reflectivity using a $\mathrm{HeNe}$ laser with wavelength $633 \mathrm{~nm}$. The laser beam reflected by the sample, is collected by a photodiode positioned outside the vacuum chamber.

After irradiation at different fluences the sheet resistance has been measured with a four point probe and HP4156B parameter analyzer. The temperature dependence has been evaluated using a Temptronics thermal chuck, in the range from $-20{ }^{\circ} \mathrm{C}$ to $30{ }^{\circ} \mathrm{C}$, in order to avoid possible annealing of the defects produced during the ion irradiation.

Raman spectra have been collected by a Horiba Jobin Yvon HR800 system equipped with a $633 \mathrm{~nm}$ HeNe laser focused through a microscope in order to have with a spot diameter of about $4 \mu \mathrm{m}$. The power of the laser was kept below $1 \mathrm{~mW}$, thus avoiding sample heating. The spectral window is $320 \mathrm{~cm}^{-1}$ and the resolution $0.2 \mathrm{~cm}^{-1}$. Each spectrum has been acquired using 3 accumulations, each with collecting time of $40 \mathrm{~s}$. Four different regions have been sampled.

Further analyses to determine the microstructure and the grain size have been conducted by transmission electron microscopy (TEM) using a JEOL 2010F operating at 
$200 \mathrm{keV}$ and equipped with a low magnification camera for electron diffraction analyses. Samples were prepared at room temperature by mechanical polishing and chemical etching to avoid any artifact due to heating and/or milling, obtaining a membrane of $50 \mathrm{~nm}$ of capped GST on a thin layer of silicon oxide.

Film structure was analyzed by XRD in glancing incidence mode $\left(0.5^{\circ}\right)$ using a Smartlab Rigaku diffractometer, equipped with a rotating anode of $\mathrm{Cu} \mathrm{K} \alpha$ radiation operating at $45 \mathrm{kV}$ and $200 \mathrm{~mA}$.

\subsection{Simulation Details}

We have carried out Density Functional Theory (DFT) simulations using the QUICKSTEP ${ }^{25}$ code included in the CP2K package. ${ }^{26}$ In this code, Kohn-Sham orbitals are expanded in a localized Gaussian-type basis set with triplezeta plus polarization quality, whereas the charge density is expanded in plane waves. We employ a charge-density cutoff of 300 Ry. We do not include spin-orbit coupling in our simulations; instead, we use scalar-relativistic Goedecker ${ }^{27}$ pseudopotentials. Spin-orbit coupling is a crucial ingredient of topological insulator phases, nevertheless it has small effects on the structural properties and the electron localization properties of strongly disordered models, ${ }^{25}$ which are the main focus of this work. The Brillouin zone is sampled at the $\Gamma$ point. We use the functionals developed by Perdew, Burke and Ernzerhof, ${ }^{26}$ which are based on the generalized gradient approximation.

\section{RESULTS AND DISCUSSION}

Figure 1 shows the normalized reflectivity as a function of the fluence, as measured during irradiation of the cubic or trigonal GST225 (blue curves) and GST124 (red curves)

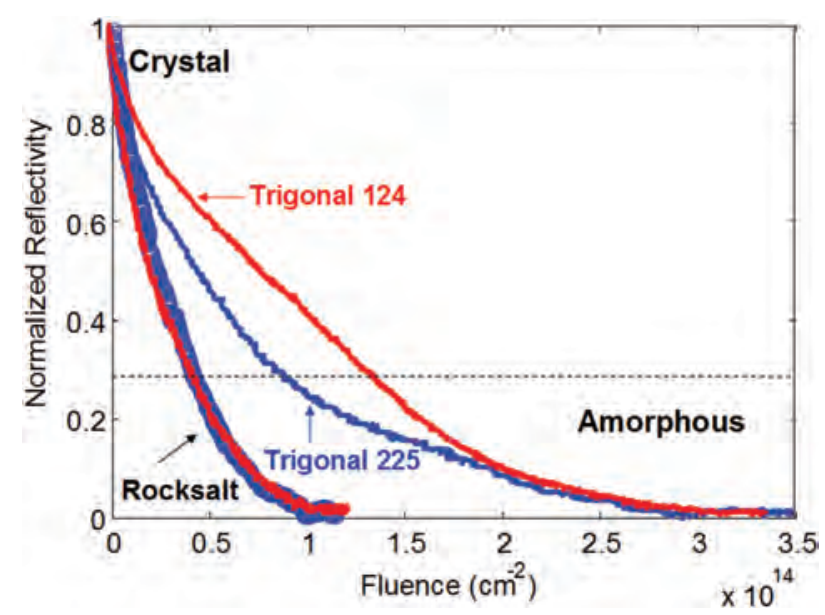

Fig. 1. Normalized reflectivity as a function of fluence, measured in situ at room temperature for GST124 (red curves) and GST225 (blue) in the rocksalt or in the trigonal phase. For the rocksalt phase the same behavior is observed, while for the trigonal structure higher fluence is required for GST124 to form an amorphous phase. The amorphization threshold is depicted by a horizontal dashed line. at room temperature. In all the samples the highest reflectivity is measured before irradiation $\left(R_{\max }\right)$ and decreases as the fluence increases, down to a minimum value $R_{\min }$, corresponding to the complete amorphization. ${ }^{28}$ The normalized reflectivity reported in Figure 1 is $\left(R-R_{\min }\right) /$ $\left(R_{\max }-R_{\min }\right)$. In both GST225 and GST124 when the irradiation is performed at $77 \mathrm{~K}$ (not shown) the amorphization threshold for the trigonal and the rocksalt phase is the same. It corresponds to the a fluence of $10^{13} \mathrm{~cm}^{-2}$ to obtain a decrease by a factor $1 / e$ in the reflectivity value. ${ }^{29}$

As expected, due to the dynamic annealing, a higher fluence is required at room temperature, since the disorder can be partly recovered by atomic rearrangement. ${ }^{30}$ However, as we have previously shown for GST225, the effect of the dynamic annealing of defects is not the same in the two crystalline structures, obtaining at room temperature an amorphization threshold of $3 \times 10^{13} \mathrm{~cm}^{-2}$ for the rocksalt phase and $8 \times 10^{13} \mathrm{~cm}^{-2}$ for the stable trigonal phase. $^{31}$

Furthermore, from Figure 1 we see that, at room temperature, the same threshold is obtained for GST124 and 225 in the rocksalt phase, whilst a different behavior is observed for the trigonal structure, with a higher fluence, equal to $1.2 \times 10^{14} \mathrm{~cm}^{-2}$, required to amorphize the GST124.

Since the two compositions exhibit the same threshold at room temperature in the rocksalt phase, again the difference seems to be peculiar to the trigonal structure.

From a pure ballistic point of view, given a chemical composition, ion trajectories and related effects, such as displacement rate, are not expected to depend on the material crystalline structures, apart from directional effects, such as planar or axial channeling, that should be avoided. As confirmed by irradiation at low temperature, these are clearly not relevant to the present study. Therefore, the different behavior observed at room temperature has to be related to the mobility of the displaced target atoms and in particular to the possible paths for annealing of defects in the trigonal structure. This structure is characterized by the presence of van der Waals gaps (vdW), separating two Te planes. We call the gaps van der Waals gaps since the typical distance of $0.28 \mathrm{~nm}$ is much larger than the average interplanar distance of $0.18 \mathrm{~nm}$ found in this phase. ${ }^{16}$ This is indicative for a very weak interplanar coupling. vdW gaps can be found at the completion of a unitary formula, made by a stacking of anionic (Te) and cationic planes (mixed Ge and Sb). This occurs each 9 or 7 planes in the GST225 or GST124 composition, respectively.

We therefore may argue that, thanks to the high atomic mobility, typical of phase change materials, and to the dilute collision cascade that prevents a direct recombination of vacancy and interstitials, the atoms displaced by the impinging ions diffuse and stop in the van der Waals gaps that act as preferential sinks.

This assumption is corroborated by the results obtained by Raman spectroscopy and by electrical measurements. 


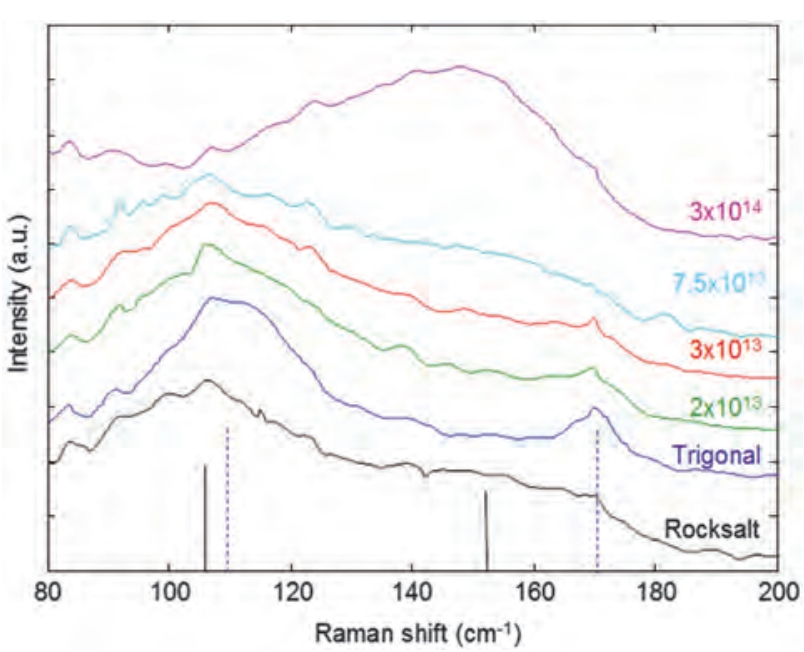

Fig. 2. Raman spectra of GST124 in the trigonal phase (blue) and after irradiation at different fluences. For comparison the Raman spectrum of the GST124 in the rocksalt phase is also reported as black line. As the fluence increases, first the $A_{1 \mathrm{~g}}$ peak at $170 \mathrm{~cm}^{-1}$ disappears (red curve), then a broad contribution at about $155 \mathrm{~cm}^{-1}$ is detected (cyan), characteristic for the rocksalt phase. At the highest fluence the spectrum becomes that of the amorphous phase (magenta).

Figure 2 shows the Raman spectra of the GST124 samples acquired ex situ after irradiation at different fluences. According to $a b$ initio calculations of the Raman peaks of the trigonal phase, the vibrations above $150 \mathrm{~cm}^{-1}$ are due to $A$-type phonons (vibrations along the stacking $c$-axis). ${ }^{32}$ Figures 3(a) and (b) show a sketch of the $E_{\mathrm{g}}$ and $A_{1 \mathrm{~g}}$ phonons, respectively, as reported in Ref. [31] for the trigonal phase. Calculated frequencies are also reported in the figure. In particular, the A mode at highest frequency $\left(A_{1 \mathrm{~g}}(4)\right)$ is modulated by the outermost Te-X bond, closer to Te-Te gaps. This peak is highest in the un-irradiated sample and decreases for increasing irradiation fluence,

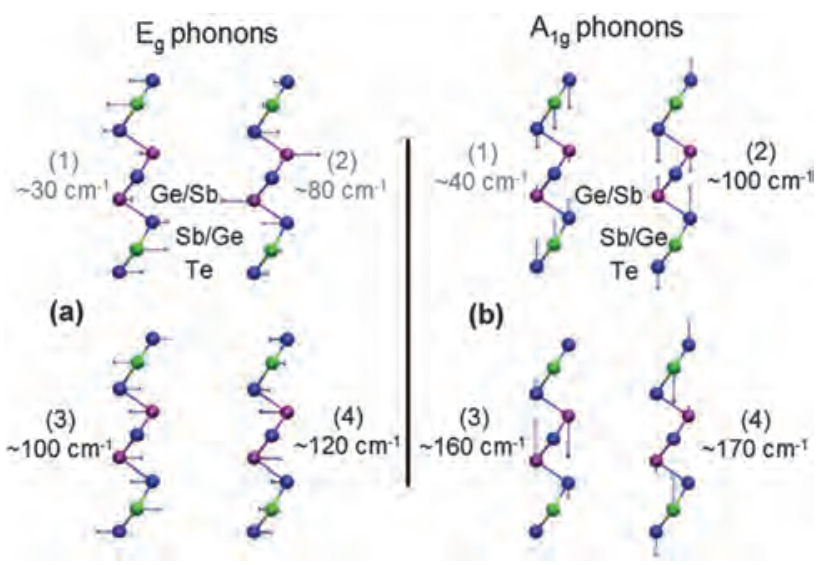

Fig. 3. Sketch of the displacement patterns of Raman active $E_{g}$ (a) and $A_{1 \mathrm{~g}}$ (b) phonons at the $\Gamma$ point, and corresponding frequencies as calculated in Ref. [30] for the trigonal phase. For $E_{\mathrm{g}}$-mode, displacements in the $a b$ planes are involved, while for the $A_{1 \mathrm{~g}}$-mode the displacements occur along the $c$ axis. completely disappearing at a fluence of $3 \times 10^{13} \mathrm{~cm}^{-2}$. The same is observed also for films with composition GST225, in which the $A_{1 \mathrm{~g}}$ peak disappears at a fluence of $2 \times 10^{13} \mathrm{~cm}^{-2}$.

The peak at $106 \mathrm{~cm}^{-1}$, characteristic of the vibrations in the basal $a b$ plane ( $E$-type modes), ${ }^{32}$ is instead clearly detectable, up to higher fluence, indicating the material is crystalline. Indeed, since in the cationic $a b$ planes perpendicular to the $c$ direction (basal planes) $\mathrm{Ge}$ and $\mathrm{Sb}$ atoms are mixed, the disorder induced by ion irradiation in these planes seems to not largely modify the Raman spectrum.

Increasing the fluence up to $3 \times 10^{14} \mathrm{~cm}^{-2}$ give rises to a Raman spectrum typical of the amorphous phase, whilst at intermediate fluences $\left(7.5 \times 10^{13} \mathrm{~cm}^{-2}\right)$ a broad contribution at about $150 \mathrm{~cm}^{-1}$ is detected, together with the peak at $106 \mathrm{~cm}^{-1}$, typical of the crystalline rocksalt phase.

Confirmation of the crystalline phases formed under irradiation have been obtained by $\mathrm{X}$ ray diffraction (XRD) analyses. Figure 4 shows the XRD spectra of the irradiated samples. The samples irradiated at lower fluences ( 2 and $3 \times 10^{13} \mathrm{~cm}^{-2}$ ) exhibit the diffraction pattern of the trigonal phase with lattice parameters $a=b=4.22 \pm 0.01 \AA$, $c=40.7 \pm 0.2 \AA$. In the case of irradiation at higher fluences the diffraction peaks correspond to the rocksalt structure with lattice parameter $a=6.06 \pm 0.01 \AA$. Peaks are larger, indicating the formation of very small grains, in agreement to what was previously observed also for GST225. ${ }^{31}$ Some residual trigonal phase is also detected.

Further insights can be obtained through the electrical measurements. As shown in Figure 5(a) the resistivity increases as a function of fluence and the temperature coefficient of resistance, evaluated from the linear fit of resistivity data versus temperature, exhibits a transition from positive to negative values. The first negative value

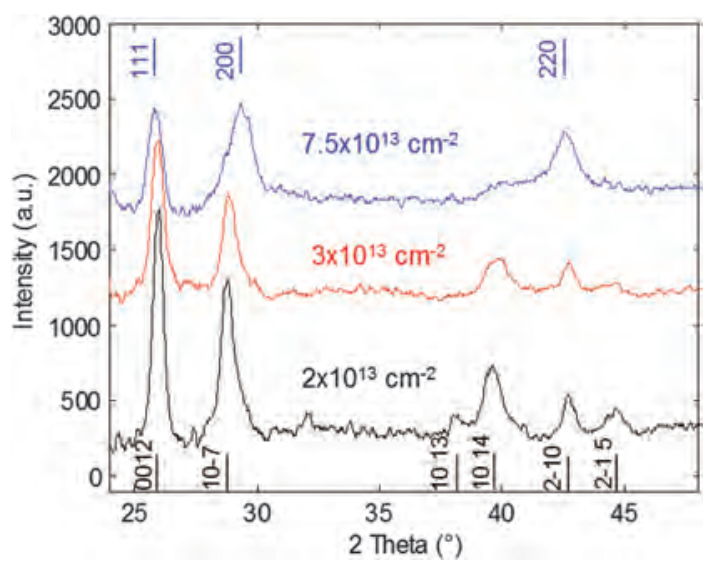

Fig. 4. X-ray diffraction spectra of GST124 samples irradiated at different fluences. At lower fluences (black and red lines) the films exhibit the diffraction pattern of the trigonal phase. On the contrary, with irradiation at a fluence of $7.5 \times 10^{13} \mathrm{~cm}^{-2}$ the film is crystalline with the rocksalt structure. 

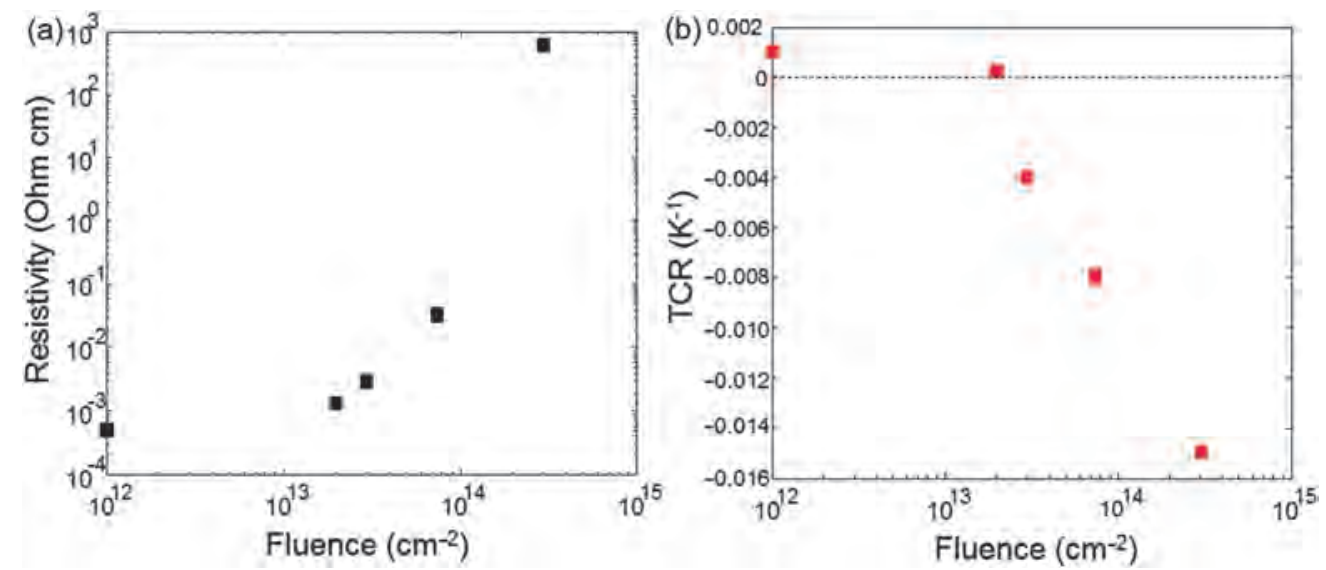

Fig. 5. (a) Resistivity and (b) temperature coefficient of resistance of GST124 as a function of fluence after irradiation at room temperature.

is obtained at a fluence of $3 \times 10^{13} \mathrm{~cm}^{-2}$. This result confirms that the metallic conduction is closely interwoven with the presence of vdW gaps or ordered vacancy layers, both characterized by the Raman peak at $170 \mathrm{~cm}^{-1}$. Whenever this peak disappears in the Raman spectrum, the conductivity behavior becomes typical of an insulating material, indicating that the vacancies are disordered and localized states are formed at the Fermi level.

The modifications induced by ion irradiations are usually described by the displacements of atoms (DPA) produced by the energy transfer. By employing the SRIM code we can obtain an estimation of the degree of disorder-in terms of displaced atoms - required to produce the observed transitions. However, the two studied compositions are very similar, and therefore the calculated DPA at a given dose is the same for GST225 and GST124, thus not explaining why the phase transitions in the trigonal structures have instead been observed at different fluences. This is because the SRIM code cannot take into account the presence of the $\mathrm{vdW}$ gaps. Indeed, if our description is correct, it is more appropriate to consider the filling of the gaps by atoms displaced by the collision cascade, instead of the displaced atoms themselves. Considering the high activation energy for migration of Te atoms and the unfavorable Te-Te antibonds, ${ }^{33}$ the probability to find displaced Te atoms inside the gaps is very low and we can only consider $\mathrm{Ge}$ and $\mathrm{Sb}$ atoms. This implies that only about half of the total displaced atoms are trapped in the gaps.

Figure 6 reports the calculated gap filling, obtained as the ratio between the Ge and Sb displaced atoms (SRIM data) and the vacancy concentration in the alloy, as a function of the irradiation fluence. The gap filling is calculated in respect to the number of available vacancies for each stoichiometry. This means that anyway, to preserve the stoichiometry, $100 \%$ of gap filling corresponds to $25 \%$ or $20 \%$ of vacancies for GST124 or GST225, respectively, as in the disordered rocksalt structure. Since the number of stoichiometric vacancies is higher for GST124, compared to GST225, lower gap filling is reached in GST124, for the same fluence, therefore explaining the higher fluences required for the transitions.

The first observed transition is the electronic metalinsulator transition. This is observed in both compositions above $30 \%$ of gap filling. According to density function theory (DFT) simulations performed for GST124, starting from the rocksalt phase, the reverse insulator to metal transition is expected to occur upon formation of depleted layers with vacancy concentrations around $75 \%$, that corresponds to gap filling of $33 \%$, in good agreement with the present findings.

By further increasing the fluence, the conversion to the rocksalt structure is observed, as shown in literature ${ }^{31}$ for GST225, and indicated by Figures 2 and 4 for GST124. This is observed experimentally at fluences corresponding to gap filling above $80 \%\left(4 \times 10^{13} \mathrm{~cm}^{-2}\right.$ and $7.5 \times 10^{13} \mathrm{~cm}^{-2}$, for GST225 and GST124, respectively), although the transition fluence cannot be strictly determined. By DFT simulations of disordered GST124 with trigonal stacking, we find that partial formation of vacancy

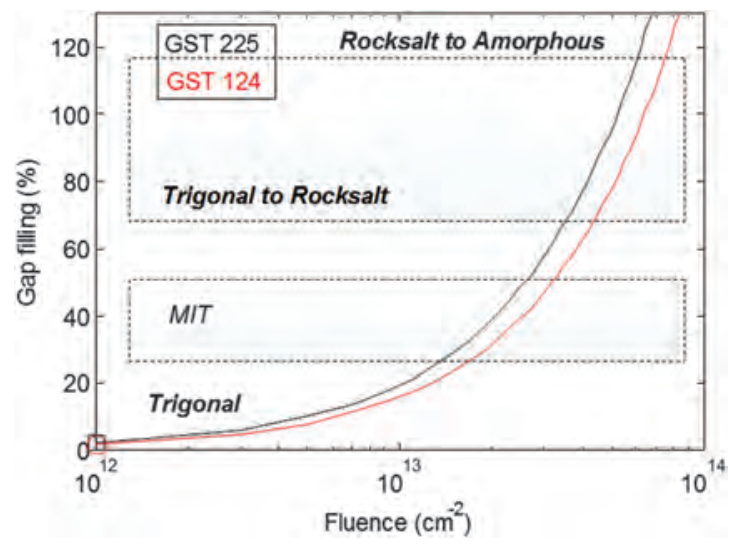

Fig. 6. Percentage of gap filling determined by using the calculated displaced atoms by SRIM and assuming that half of these are trapped into the van der Waals gaps. Dashed rectangular regions indicate the approximate ranges of occurrence of the observed phase transitions. 


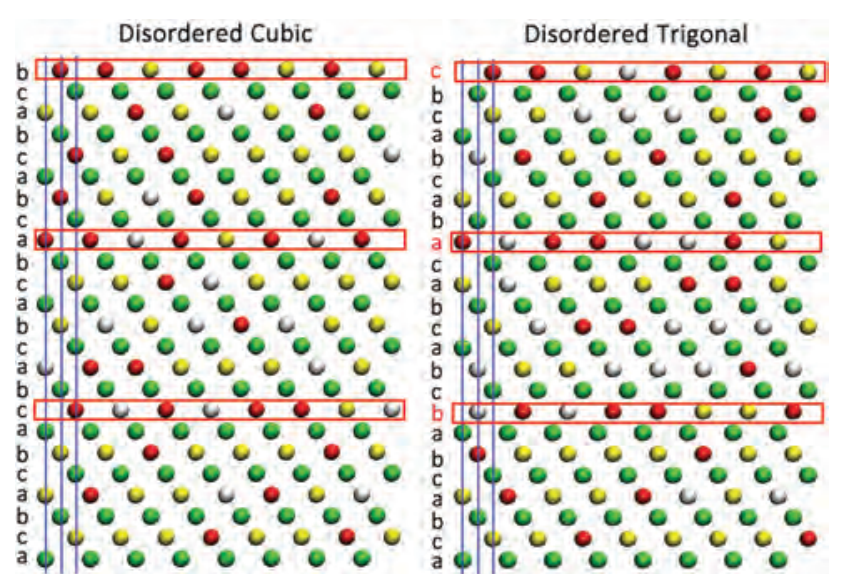

Fig. 7. Models of disordered cubic and trigonal GST124. The vacancy concentration is equal to $50 \%$ for both models (66.7\% of gap filling). $\mathrm{Ge}, \mathrm{Sb}$, and Te atoms are rendered with gray, yellow, and green spheres, whereas atomic vacancies are rendered with red spheres. The vacancy layers are marked with orange boxes.

layers (with 50\% vacancy concentration), corresponding to $66.7 \%$ of the gap filling, makes the trigonal phase less energetically favorable than the corresponding models with cubic stacking (see Fig. 7). The energy difference is 13 and $15 \mathrm{meV} /$ atom for GST124 and GST225, respectively. It is difficult to estimate from the DFT simulations the critical filling at which the hexagonal-cubic transition occurs. However, we have shown that at $33 \%$ of gap filling the hexagonal phase is still more favorable, ${ }^{19}$ therefore we can estimate that the critical theoretical value lies between $66 \%$ and $100 \%$ of gap filling. $100 \%$ of gap filling for GST124 still corresponds to an amount of $25 \%$ of stoichiometric vacancies, which is the case of the completely disordered rocksalt structure. It is worth to note that very small grains (about $4 \mathrm{~nm}$ in size) with rocksalt structure start to form within large grains with trigonal structure. ${ }^{31}$ Since in order to clearly detect the rocksalt phase, for example by X-ray diffraction, a sufficient large volume fraction is needed, the value of gap filling at which this transition occurs could be experimentally overestimated. Indeed, we obtain an XRD pattern corresponding to the rocksalt phase at a fluence of $7.5 \times 10^{13} \mathrm{~cm}^{-2}$, that corresponds to more than $100 \%$ of gap filling.

Above $100 \%$ gap filling the disorder becomes higher than in the disordered rocksalt phase and the film also start to become amorphous.

As a guide, we have drawn in Figure 6 dashed rectangular regions to indicate the approximate ranges of occurrence of the observed phase transitions. These are not sharp transitions but, nevertheless, the XRD data clearly indicate the existence of a range of fluences at which the film is mainly crystalline (possibly with some amorphous inclusions) but has changed structure from the trigonal to the rocksalt phase, since in this metastable structure a higher amount of disorder can be accommodated.

\section{CONCLUSIONS}

We have studied the strain development and damage accumulation induced by ion irradiation on polycrystalline GST thin films with composition GST124 and GST225. At low temperature $(77 \mathrm{~K})$ the two compositions and crystalline structures exhibit the same behavior. At room temperature, in the rocksalt phase, GST225 and GST124 exhibit the same amorphization threshold, while in the trigonal structures higher fluences are required to amorphize the GST124. This has been explained as an effect of dynamic annealing in the peculiar crystalline structure, utilized in the trigonal phase, which is characterized by the presence of van der Waals gaps. These act as preferential sinks for the diffusion of the displaced atoms. The filling of these gaps tunes the electronic and structural properties. Several phase transitions are observed in the trigonal phase under ion irradiation, which are all well explained by the filling of vdW gaps. The first modification is the electronic transition from metallic to insulating behavior, corresponding to the filling of more than $30 \%$ of the gaps. This compromises the metallic conduction, due to the formation of localized states at the Fermi level. By further increasing the disorder, and thus filling the gaps above $70 \%$, the films convert into the metastable rocksalt phase. The high energy penalty of vacancy disorder in the trigonal phase provides the driving force for the inverse trigonal to cubic structural transition, as evidenced by the DFT simulations.

Acknowledgments: The authors thank Bio-nanotech Research and Innovation Tower (BRIT) laboratory of University of Catania for the diffractometer facility.

\section{References and Notes}

1. M. Nastasi and J. W. Mayer, Ion Implantation and Synthesis of Materials, Springer-Verlag, Berlin, Heidelberg (2006).

2. M. Wuttig and N. Yamada, Nature Materials 6, 824 (2007).

3. S. R. Ovshinsky, Phys. Rev. Lett. 21, 1450 (1968).

4. G. W. Burr, M. J. Breitwisch, M. Franceschini, D. Garetto, K. Gopalakrishnan, B. Jackson, B. Kurdi, C. Lam, L. A. Lasras, A. Padilla, B. Rajendran, S. Raoux, and R. S. Shenoy, J. Vac. Sci. Technol. B 28, 223 (2010).

5. S. Lai and T. Lowrey, IEDM Technical Digest, International, Washington, DC, USA (2001), pp. 36.5.1-36.5.4.

6. A. Redaelli and A. Pirovano, Nanotechnology 22, 254021 (2011).

7. N. Yamada, E. Ohno, K. Nishiuchi, N. Akahira, and M. Takao, J. Appl. Phys. 69, 2849 (1991).

8. B. J. Kooi and J. Th. M. De Hosson, J. Appl. Phys. 92, 3584 (2002).

9. T. Matsunaga, N. Yamada, and Y. Kubota, Acta Crystallogr. B Struct. Sci. Cryst. Eng. Mater. 60, 685 (2004).

10. T. Matsunaga, R. Kojima, N. Yamada, K. Kifune, Y. Kubota, Y. Tabata, and M. Takata, Inorganic Chemistry 45, 2235 (2006).

11. K. S. Siegert, F. Lange, R. Sittner, H. Volker, C. Schlockermann, T. Siegrist, and M. Wuttig, Reports on Progress in Physics 78, 013001 (2015).

12. R. H. S. Winterton, Contemporary Physics 11, 559 (1970).

13. I. I. Petrov, R. M. Imamov, and Z. G. Pinsker, Sov. Phys. Crystallogr. 13, 339 (1968). 
14. J. Momand, R. Wang, J. E. Boschker, M. A. Verheijen, R. Calarco, and B. J. Kooi, Nanoscale 7, 19136 (2015).

15. R. Wang, V. Bragaglia, J. E. Boschker, and R. Calarco, Cryst. Growth Des. 16, 3596 (2016).

16. A. M. Mio, S. M. S. Privitera, V. Bragaglia, F. Arciprete, C. Bongiorno, R. Calarco, and E. Rimini, Nanotechnology 28, 065706 (2017).

17. T. Siegrist, P. Jost, H. Volker, M. Woda, P. Merkelbach, C. Schlockermann, and M. Wuttig, Nat. Mater. 10, 202 (2011).

18. N. P. Breznay, H. Volker, A. Palevski, R. Mazzarello, A. Kapitulnik, and M. Wuttig, Phys. Rev. B 86, 205302 (2012).

19. W. Zhang, A. Thiess, P. Zalden, R. Zeller, P. H. Dederichs, J.-Y. Raty, M. Wuttig, S. Blügel, and R. Mazzarello, Nat. Mater. 11, 952 (2012).

20. V. Bragaglia, F. Arciprete, W. Zhang, A. M. Mio, E. Zallo, K. Perumal, A. Giussani, S. Cecchi, J. Boschker, H. Riechert, S. Privitera, E. Rimini, R. Mazzarello, and R. Calarco, Sci. Rep. 6, 23843 (2016).

21. K. S. Siegert, F. R. L. Lange, E. R. Sittner, H. Volker, C. Schlockermann, T. Siegrist, and M. Wuttig, Rep. Prog. Phys. 78, 013001 (2015).

22. M. Kumar, A. Vora-Ud, T. Seetawan, and J. G. Han, Energy Technol. 3, 1 (2015)
23. S. Privitera, G. D'Arrigo, A. M. Mio, N. Piluso, F. La Via, and E. Rimini, IEEE Trans. Elec. Dev. 61, 2879 (2014).

24. R. Simpson, P. Fons, A. V. Kolobov, T. Fukaya, M. Krbal, T. Yagi, and J. Tominaga, Nat. Nanotech. 6, 501 (2011).

25. J. VandeVondele, M. Krack, F. Mohamed, M. Parrinello, T. Chassaing, and J. Hutter, Comput. Phys. Comm. 167, 103 (2005)

26. J. Hutter, M. Iannuzzi, F. Schiffmann, and J. VandeVondele, WIREs Comput. Mol. Sci. 4, 15 (2014).

27. S. Goedecker, M. Teter, and J. Hutter, Phys. Rev. B 54, 1703 (1996)

28. N. Yamada, E. Ohno, K. Nishiuchi, N. Akahira, and M. Takao, J. App. Phys. 69, 2849 (1991).

29. S. Privitera, A. M. Mio, J. Benke, C. Persch, E. Smecca, A. Alberti, and E. Rimini, MRS Advances 1, 2701 (2016).

30. P. J. Schultz, C. Jagadish, M. C. Ridgway, R. G. Elliman, and J. S. Williams, Phys. Rev. B 44, 9118(R) (1991).

31. S. Privitera, A. M. Mio, E. Smecca, A. Alberti, W. Zhang, R. Mazzarello, J. Benke, C. Persch, F. La Via, and E. Rimini, Phys. Rev. B 94, 094103 (2016).

32. G. C. Sosso, S. Caravati, C. Gatti, S. Assoni, and M. Bernasconi, J. Phys. Condens. Matter 21, 245401 (2009).

33. B. Zhang, W. Zhang, Z.-J. Shen, Y.-J. Chen, J.-X. Li, S.-B. Zhang, Z. Zhang, M. Wuttig, R. Mazzarello, E. Ma, and X.-D. Han, Appl. Phys. Lett. 108, 191902 (2016).

Received: 19 December 2016. Accepted: 3 May 2017. 